\title{
Dysphagia and drooling in parkinson disease improved by sensory cues
}

\author{
Galli J ${ }^{1}$, Marchese $\mathrm{MR}^{2 *}$, De Canio $\mathrm{C}^{3}$, Battaglia $\mathrm{M}^{3}$, Gatto $\mathrm{G}^{4}$, Santandrea $\mathrm{L}^{4}$ and Paludetti $\mathrm{G}^{1}$ \\ ${ }^{1}$ Department of Aging, Neuroscience, Orthopedics and Head and Neck Sciences, UOC of Otorhinolaryngology, Istituto di Otorinolaringoiatria "Fondazione \\ Policlinico Univeristario A. Gemelli IRCCS - Università Cattolica del Sacro Cuore”, Roma, Italy \\ ${ }^{2}$ Department of Aging, Neuroscience, Orthopedics and Head and Neck Sciences, UOC of Otorhinolaryngology, "Fondazione Policlinico Universitario A. Gemelli \\ IRCCS”, Roma, Italy \\ ${ }^{3}$ ENT Department, San Carlo Hospital, Potenza, Italy \\ ${ }^{4}$ Department of Physical Medicine and Rehabilitation, San Carlo Hospital, Potenza, Italy
}

\begin{abstract}
In the Parkinson disease (PD) ones of the most common motor and non-motor symptoms are respectively dysphagia and drooling. The usual management includes swallowing rehabilitation maneuvers especially for the oral and pharyngeal phases. Basing on the role of sensory cues demonstrated for gait and dysarthria we describe two cases of PD with dysphagia symptoms and poor saliva control who were subjected to rehabilitation therapy combined with visual and auditory cues. The dysphagia-related impairments have a direct influence on the nutritional and health status of the patients and are associated with increased morbidity and mortality. The results observed at the early and late controls were encouraging and promote the research about the role of sensory cues in enhance the efficacy of the physical rehabilitation.
\end{abstract}

\section{Introduction}

Oral motor disorders such as dysphagia, drooling and dysarthria are common in patients affected by PD [1]. 75-97\% of cases will suffer from dysphagia [2-4] reflecting the underlying motor impairments and the extent of the disease's progression, whereas drooling is reported by about one-quarter to one-half of the population, depending on the diagnostic criterion [5]. The dysphagia has a direct influence on the nutritional and health status of the patients, and it is associated with increased morbidity and mortality. The exercises of tongue strengthening, and tongue control were frequently indicated because tongue movements of PD patients, especially those responsible for propulsion and chewing, are considered hypokinetic in the oral phase that is usually longer and even slower than the pharyngeal phase. To date the PD dysphagia management is based on traditional compensatory and rehabilitation maneuvers [6] whose results are not unanimous. Other PD motor problems, such as gait control, are actually treated with new rehabilitation technique. Particularly auditory and visual cueing methods are commonly applied to evoke a more goal-directed type of motor control by inducing a re-direction from more to less affected neural circuits [7]. The positive effects of cueing are abundant and well known [8-10]. Visual cues, which are spatial in nature, mainly seem to correct and regulate the scaling and amplitude generation during the movement whereas temporal auditory cues generally affect the timing of action and coordination [8]. We introduced the sensory cues in traditional rehabilitation protocol of $\mathrm{PD}$ dysphagia with the purpose to evaluate the effect on the swallowing function and drooling.

\section{Cases report}

Two men 61 and 68 years-old respectively, affected by longstanding Parkinson disease (PD) (stage 3 of the Hoehn and Yahr scale) were referred. They suffered from drooling and swallowing related symptoms in the on-phase and were not previously treated. Both cases complained severe sensations of food stopping in the mouth and sticking in the throat.

Physical examination revealed impairment of tongue movements (protrusion, lateralization, tip elevation, intraoral circular movements) and lip performance (pop). Moreover, the fiberoptic endoscopic assessment (FEES) showed deficit of oral bolus propulsion, abnormal pharyngeal squeeze and post-swallow residue in the pharynx. Both patients were unable to perform vocal diadochokinetic exercises (repeating the /pa/, / ta/ and / ka/ syllables, of the tri-syllabic/pataka/ sequence, for five seconds uninterruptedly). The swallowing symptoms and saliva control were evaluated on the basis of patients' self-reports by administering the Radboud Oral Motor inventory for Parkinsons's disease (ROMP) [9-11] (filling out with the help of their caregivers). Before the treatment the scores for swallowing and drooling subscales were respectively 25 and 36 in case 1 and 28 and 39 in case 2 .

The patients were treated in the outpatient ENT department. The therapy setting consisted of one-hour session twice a week for 4 weeks. A written informed consent was obtained from both the participants included in the study.

*Correspondence to: Maria Raffaella Marchese, Department of Aging, Neuroscience, Orthopedics and Head and Neck Sciences, UOC of Otorhinolaryngology, "Policlinico A. Gemelli" Foundation, L.go "A. Gemelli", 8 - 00168 Rome - Italy, E-mail: mariaraffaella.marchese@policlinicogemelli.it

Key words: dysphagia, parkinson, sensory cues

Received: April 24, 2020; Accepted: April 30, 2020; Published: May 05, 2020 
During each session were provided the rehabilitation maneuvers finalized to increase lip sensitivity, strength of labial muscles, tongue control, velar-pharyngeal competence and the activity of pharyngeal muscles. The exercises for tongue control e labial continence were performed with combined auditory, visual and tactile cues. The auditory cue was obtained by employing a metronome set at individual cadence adequately adapted over time. The visual cue consisted of to show the opening the hand of the side in which move the tongue. Finally, the tactile cue was administered, during the exercises of intraoral rotation of the tongue, by touching the lips clockwise and counter-clockwise along an ideal line that mimed the intraoral tongue movements. Homework was given and they were instructed to the compensatory effortful swallow [12] and chin-tuck maneuvers [13] in order to improve pharyngeal strength and airway protection respectively.

At the end of therapy subjectively the sensations of food stopping in the mouth and sticking in the throat decreased. Physical assessment showed improvement of the tongue and lip movements. The patients became able to perform diadochokinetic exercises. The FEES showed almost normal oral bolus propulsion, effective pharyngeal squeeze and absence of pathologic post-swallow residues in the pharynx. After two weeks the scores for swallowing and drooling subscale were respectively 18 and 20 in case 1 and 14 and 21 in case 2 . The ROMP results remained stable at the end of the therapy.

\section{Discussion}

In the conditions of the PD itself, such as rigidity and bradykinesia, dysphagia and drooling are commonly observed. Particularly the impairement in swallowing may be caused by a change of the oral or pharyngeal phase. Pneumonia prevention makes necessary for PD patients to attend rehabilitation programs whose goal is to obtain better strength and mobility of the involved structures. The exercises of the lip and tongue are frequently indicated because their movements are considered hypokinetic. Generally, behavioral strategies are suggested to shift habitual motor control to a goal-directed one by specific self-prompting instructions or cues or a combination of these. The beneficial effects of the cues are attributed to a re-direction from more to less affected neural circuits [7]. The positive effects of cueing in PD patients are abundant and known [8]. Regarding head-neck district was demonstrated that topic cues increase intelligibility in dysarthria [14] and that, as showed by Pinnington et al. [15], verbal cueing resulted in a significant reduction in the duration of the oral phase of swallowing. Moreover, Nakamura and Satoshi [16] observed that auditory verbal cues can improve the swallow physiology in normal participants. In order to strength the effectiveness of tongue and labial continence rehabilitation maneuvers we combined auditory non-verbal and visual cues. To date no work has addressed the effects of these last cues in dysphagic PD patients. We obtained encouraging subjective and physical results in both saliva control and swallowing quality function. It is probably that the visual cues correct and regulate the scaling and amplitude generation during movements and on the other hand the auditory cues affect the timing and coordination of lip and tongue performances as occurs for the gait control [8]. Nevertheless, further studies need to define the role of the cues in PD dysphagia first of all by increasing the study sample and comparing results of patients treated with traditional rehabilitation maneuvers with the ones obtained by the association with sensory cues.

\section{Conclusion}

In conclusion we observed improvement of swallowing function and saliva control in patients affected by Parkinson disease by adding sensory cues to traditional rehabilitation exercises. These results may encourage to further studies.

\section{References}

1. Bloem BR, Kalf JG, van de Kerkhof PC (2009) Debilitating consequences of drooling J Neurol 256: 1382-1383.

2. Logemann JA, Blonsky ER, Boshes B (1975) Editorial: dysphagia in parkinsonism JAMA 231: 69-70.

3. Stroudley J, Walsh M (1991) Radiological assessment of dysphagia in Parkinson's disease. Br J Radiol 64: 890-893.

4. Bird MR, Woodward MC, Gibson EM (1994) Asymptomatic swallowing disorders in elderly patients with Parkinson's disease: a description of findings on clinical examination and videofluoroscopy in sixteen patients. Age Ageing 23: 251-254.

5. Goetz CG, Tilley BC, Shaftman SR (2008) Movement Disorder Society-sponsored revision of the Unified Parkinson's Disease Rating Scale (MDS-UPDRS): scale presentation and clinimetric testing results. Mov Disord 23: 2129-2170.

6. Crary MA (2009) Treatment for adults. In: Crary MA, Groher ME, editors. Dysphagia: clinical management in adults and children. St. Louis: Elsevier/Mosby 275-307.

7. Redgrave P, Rodriguez M, Smith Y (2010) Goal-directed and habitual control in the basal ganglia: implications for Parkinson's disease. Nat Rev Neurosci 11: 760-772.

8. Spaulding SJ, Barber B, Colby M (2013) Cueing and gait improvement among people with Parkinson's disease: a meta-analysis. Arch Phys Med Rehabil 94: 562-570.

9. Cassimatis C, Liu KP, Fahey P, Bissett M (2016) The effectiveness of external sensory cues in improving functional performance in individuals with Parkinson's disease: a systematic review with meta-analysis. Int J Rehabil Res 39: 211-218.

10. Nackaerts E, Vervoort G, Heremans E (2013) Relearning of writing skills in Parkinson's disease: a literature review influential factors and optimal strategies. Neurosci Biobehav Rev 37: 349-357.

11. Kalf JG, Borm GF, de Swart BJ (2011) Reproducibility and validity of patient-rated assessment of speech, swallowing, and saliva control in Parkinson's disease. Arch Phys Med Rehabil 92: 1152-1158.

12. Felix VN, Corrêa SMA, Soares RJ (2008) A therapeutic maneuver for oropharyngeal dysphagia in patients with Parkinson disease. Clinics 63: 661-666.

13. Luchesi KF, Kitamura S, Mourão LF (2015) Dysphagia progression and swallowing management in Parkinson's disease: an observational study. Braz J Otorhinolaryngol 81: 24-30.

14. Beukelman DR, Fager S, Hanson CUE (2002) The impact of speech supplementation and clear speech on the intelligibility and speaking rate of people with traumatic brain injury. J Med Speech- Lang Pathol 10: 237-242.

15. Pinnington LL, Muhiddin KA, Ellis RE (2000) Non- invasive assessment of swallowing and respiration in Parkin- son's disease. J Neurol 247: 773-777.

16. Nakamura A, Imaizumi S (2013) Auditory verbal cues alter the perceived flavor of beverages and ease of swallowing: a psychometric and electrophysiological analysis. Biomed Res Int 13: 892030.

Copyright: (C2020 Galli J. This is an open-access article distributed under the terms of the Creative Commons Attribution License, which permits unrestricted use, distribution, and reproduction in any medium, provided the original author and source are credited. 